\title{
Reliable cooling of GridPix detectors for cryogenic applications
}

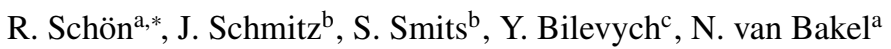 \\ ${ }^{a}$ National institute for subatomic physics Nikhef, Science Park 105, 1098XG Amsterdam, The Netherlands \\ ${ }^{b}$ MESA + Institute for Nanotechnology, University of Twente, P.O. Box 217, 7500AE Enschede, The Netherlands \\ ${ }^{c}$ Physics Institute, University of Bonn, Nußallee 12, 53115 Bonn, Germany
}

\begin{abstract}
In this article we present thermal cycling experiments of GridPix radiation imaging detectors, in view of a potential application in a cryogenic experiment. The robustness of the GridPix detector is studied for various grid designs, as well as various mechanical and thermal surroundings. The results show that a low cooling rate as well as careful thermal contact are crucial for the durability of the grid; and that the SU-8 support needs additional fixation to the chip substrate. We read out the signals of different modified layouts in an $\mathrm{Ar} / \mathrm{i}_{4} \mathrm{H}_{10}$ 90/10 atmosphere under ${ }^{55} \mathrm{Fe}$ irradiation. The energy resolution gets worse for added SU-8 support within the readout area.
\end{abstract}

Keywords: GridPix; SU-8; micro-pattern gaseous detector; InGrid; Timepix; liquid xenon; thermal expansion; cryogenics

\section{Introduction}

The GridPix detector is a type of micro-pattern gaseous de- ${ }_{31}$ tector produced by microfabricating a Micromegas grid on top ${ }_{32}$ of a 2D pixel readout chip such as Medipix or Timepix [1, 2, 3, 33 [4]. It offers high-precision imaging of ionizing radiation in two ${ }_{34}$ or three dimensions. Micromegas fabrication with microtech- ${ }_{35}$ nology offers high geometrical precision, high-purity materials ${ }_{36}$ and the possibility to mass-produce in existing clean room fa- ${ }_{37}$ cilities [5, 6]. In recent years the GridPix detector has come ${ }_{38}$ under consideration for WIMP search experiments involving ${ }_{39}$ dual-phase noble gas detectors within the R\&D programme of ${ }_{40}$ the DARWIN (dark matter WIMP search with noble liquids) consortium [7, 8].

To establish the viability of the GridPix option in these ex- ${ }^{42}$ periments, three questions are to be answered: can the detector ${ }^{43}$ be cooled down repetitively without degrading its mechanical ${ }_{45}^{44}$ properties; can we reach sufficient gas gain to detect single elec- ${ }^{45}$ trons; and can the electronics operate at cryogenic temperatures ${ }^{46}$ of $T_{\ell \mathrm{Ar}}=-186{ }^{\circ} \mathrm{C}$ and $T_{\ell \mathrm{Xe}}=-110{ }^{\circ} \mathrm{C}$ ? In this article we ad- ${ }^{47}$ dress the first question. For a discussion of the third question ${ }^{48}$ we refer to [9]. We present experimental studies on thermal ${ }^{49}$ cycles of a variety of GridPix prototypes, embedded in various ${ }^{50}$ thermal environments, and show finite-element-method (FEM) ${ }^{51}$ simulations of the mechanical behaviour of the detector during ${ }^{52}$ cooldown.

\section{Limitations of GridPix at low temperatures}

The GridPix detector consists of a silicon microchip fabri- ${ }^{57}$ cated in standard $0.25 \mu \mathrm{m}$ CMOS, a few-micrometer thick layer ${ }^{58}$

\footnotetext{
${ }^{*}$ Corresponding author

Email address: rolfs@nikhef .nl (R. Schön)
}

29 of high-ohmic material (silicon-rich silicon nitride, SiRN), insulating support pillars made of SU-8 photoresist material measuring $50 \mu \mathrm{m}$ in height, and a $1 \mu \mathrm{m}$ thick grid made of pure aluminium with holes of $38 \mu \mathrm{m}$ diameter. In the central region of the GridPix structure, a periodic pattern of pillars physically connects the grid with the microchip. At the edges, the grid should be better confined to avoid curling and to suppress sparks due to local field enhancement. Large rectangular SU-8 support ridges are normally positioned along the edges for that reason. It is however necessary to make periodic interruptions in these support ridges, so-called strain relief gaps, to prevent rupture of the SU-8 material during fabrication [10].

In previous works, the adhesion strength of this material stack was studied under various fabrication conditions and after storage in high humidity ambient [11]. The study showed a clear moisture sensitivity and a strong dependence of adhesion on the details in the fabrication process.

It can be expected that a GridPix detector with poor adhesion properties in the layer stack will delaminate upon thermal cycling, as a result of the different thermal expansion coefficients of the used materials. A preliminary test of operating a GridPix in the test cryostat of the Argon Dark Matter (ArDM) experiment confirms this. After the exposure to dual-phase argon at $-186^{\circ} \mathrm{C}$ the grid showed severe damage, see figure 1 . The ruptures in the grid start at the strain relief gaps of the SU-8 edge support that are fixed to the substrate by Globtop [7].

The robustness of GridPix detectors against thermal cycling was tested by controlled cooling with liquid nitrogen to $-130{ }^{\circ} \mathrm{C}$. This temperature is close to $-110^{\circ} \mathrm{C}$, the boiling point of xenon at atmospheric pressure which offers a less harsh environment in terms of cryogenics. First the samples were dried in a vessel in view of the moisture sensitivity mentioned above. The vessel was flushed with $\mathrm{N}_{2}$ for 12 hours at room temperature; then heated to $80^{\circ} \mathrm{C}$; then, after 30 minutes at $80^{\circ} \mathrm{C}$, 


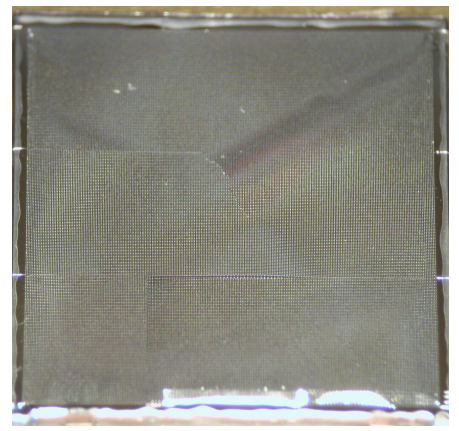

Figure 1: Picture of the damaged grid after operating a GridPix inside the test cryostat of the ArDM experiment. During the cooldown of the argon gas inside the cryostat to $-186^{\circ} \mathrm{C}$ the detector was still working as expected. It was not until the filling the lower cryostat with liquid argon that the data suggested a breakage of the grid. The picture was taken after warming up and opening the cryostat.

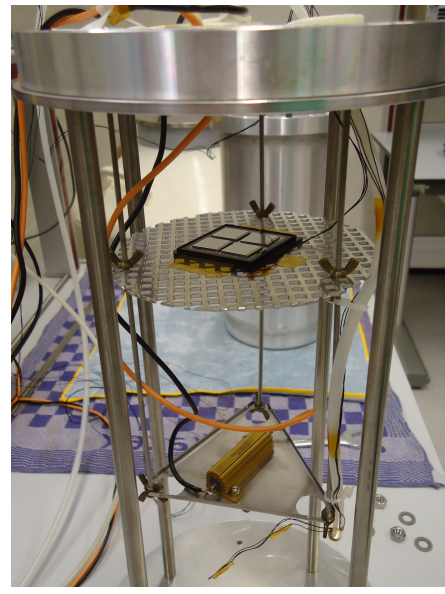

Figure 2: Picture of the inside of the test vessel. The samples rest above the heating element that controls the temperature inside the vessel. A gas tube ${ }^{87}$ provides a constant flow of dry nitrogen to purge humidity. Two temperature 88 sensors monitor the temperature of the samples and the vessel bottom.

cooled down and flushed in $\mathrm{N}_{2}$ for several hours at room tem- ${ }_{92}$ perature.

The vessel was then inserted into a dewar with liquid nitro- ${ }_{94}$ gen, well above the liquid surface. This provides cooling at $\mathrm{a}_{95}$ rate controlled to about $1 \mathrm{~K} / \mathrm{min}$ by a heating element inside the ${ }_{96}$ vessel. The setup further includes thermometers near the sam- ${ }_{97}$ ples and at the vessel bottom, see figure 2 The vessel is cooled ${ }_{98}$ to $-130^{\circ} \mathrm{C}$ and kept for 30 minutes at this temperature before ${ }_{99}$ warming it up gradually to room temperature.

The grids of two out of four devices survived the thermal cy-101 cle, figure 3a shows one of them. The two other grids show 102 a similar damage: two adjacent segments of the support ridge ${ }_{103}$ lifted off the protection layer, see figure $3 \mathrm{~b}$ This is the result ${ }_{104}$ of the stress culminating at the borders as mentioned above. $\mathrm{It}_{105}$ points to an increased stress between the SU-8 and the $\mathrm{SiRN}_{106}$ layer in regions where the SU-8 structure is large. On the other ${ }_{107}$ hand, the two surviving samples indicate that a GridPix appli- ${ }_{108}$ cation in this cold environment is technically within reach. $\quad{ }_{109}$

To monitor the situation of the samples during the cooling ${ }_{110}$ sequence the setup includes a CCD camera in further tests. ${ }_{111}$

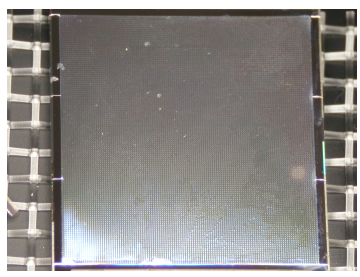

(a) Undamaged grid.

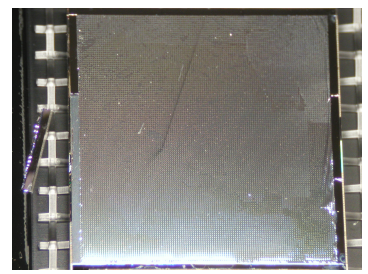

(b) Delaminated SU-8 structures.
Figure 3: Pictures of two of four GridPix chips subjected to a controlled thermal cycle down to $-130^{\circ} \mathrm{C}$. After the cycle two of the devices were still intact (a), on the other two pieces of the SU-8 support edges delaminated (b), ripping the grid.

Table 1: Important thermal and mechanical properties of all employed materials.

\begin{tabular}{lcccc}
\hline Property & $\mathrm{Si}$ & $\mathrm{SiRN}$ & $\mathrm{SU}-8$ & $\mathrm{Al}$ \\
\hline layer thickness $(\mu \mathrm{m})$ & 700 & $2 \ldots 8$ & 50 & 1 \\
\hline therm. conductivity $(\mathrm{W} /(\mathrm{mK}))$ & 105 & 35 & 0.2 & 210 \\
density $\left(\mathrm{g} / \mathrm{cm}^{3}\right)$ & 2.33 & 3.2 & 1.19 & 2.7 \\
Young's modulus $\left(10^{9} \mathrm{~N} / \mathrm{m}^{2}\right)$ & 112 & 300 & 4.02 & 68 \\
Poisson ratio () & 0.28 & 0.26 & 0.22 & 0.36 \\
CTE $\left(10^{-6} / \mathrm{K}\right)$ & 2.49 & 3.2 & 30 & 24 \\
specific heat $(\mathrm{J} /(\mathrm{kgK}))$ & 750 & 170 & 1200 & 900 \\
\hline
\end{tabular}

\section{SU-8 pattern modifications}

Finite-element simulations were conducted to investigate and visualize the effects of temperature cycling on the GridPix configuration. The thermal and mechanical properties of all employed materials as well as the thickness of the layers are listed in table 1. We can assume that the silicon substrate imposes its properties on the whole configuration thanks to its superior thickness. As clear from the table, the thermal expansion mismatch is highest at the SiRN-SU-8 interface, so this is a likely plane for delamination. A typical resulting image is shown in figure 4 All thermal expansion mismatch tends to culminate at the edges, leading to a tendency of the aluminium to rupture starting from the edges, as observed in the aforementioned tests.

As a means to prevent the observed rupture from the edges, several designs were made with different SU-8 features at the grid edges for support. The designs are sketched in figure 5 Further, bar structures were also designed into the central region, aiming to form a more rigid construction which could lead to less aluminium excursion at the edges, see figure 6

One Timepix wafer with a diameter of 8 " $(200 \mathrm{~mm})$ usually contains 107 dies of $1.6 \mathrm{~cm}$ by $1.4 \mathrm{~cm}$. We employ the same dimensions for the dummy devices not only to obtain representative results for the final goal, but also to facilitate an easy transfer of the mask to a real Timepix wafer once the best design is identified. There are 8 reference cells of the current GridPix design in the wafer layout [11]. The 11 modified designs are equally distributed over the remaining 99 fields available on the $200 \mathrm{~mm}$ wafer yielding 9 devices per new design per wafer. 


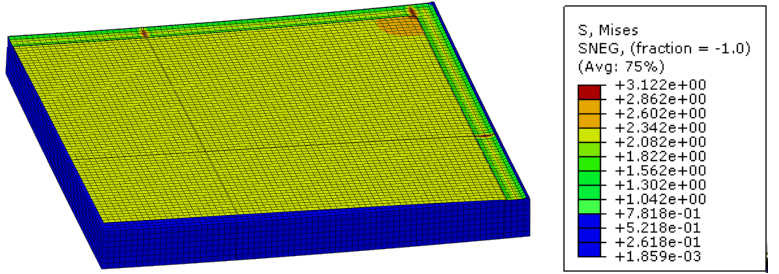

(a) Stress on ridges.
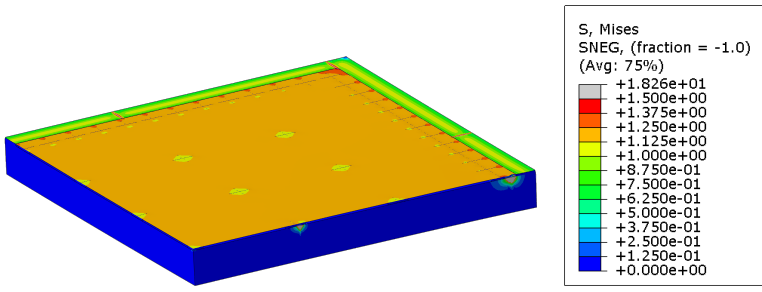

(b) Stress on grid reduced by cross support.

Figure 4: FEM simulation results showing the van Mises stress as a result of temperature decrease. The stress of shrinking aluminium grid culminates on the edges, particularly at the strain relief gaps of the SU-8 ridges (a). Introducing cross structures locally reduces the stress exerted by the grid (b). Hindering a translation of the grid reduces the stress on the grid edges. Note the different colour scale.

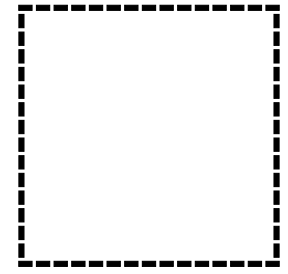

(a) Short segments.

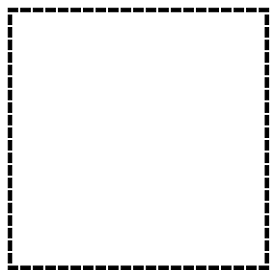

(b) Even shorter and thinner segments.

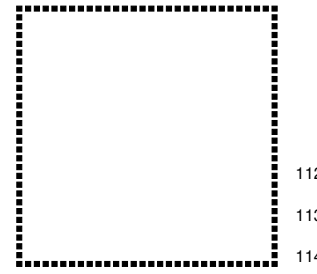

(c) Square segments. 11

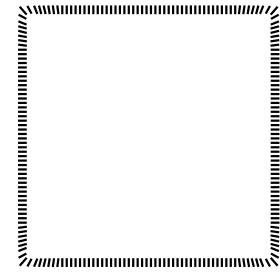

(d) Radial segments.

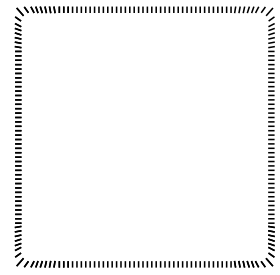

(e) Finer radial segments.
Figure 5: Simplified sketches of SU-8 ridge structures reducing the lateral stress ${ }^{124}$ along the chip's edges.

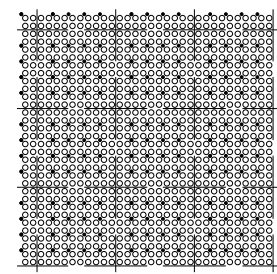

(a) Large crosses.

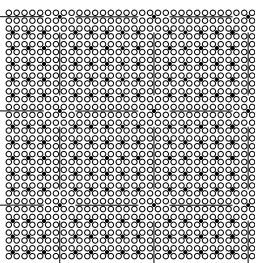

(c) Long lines.

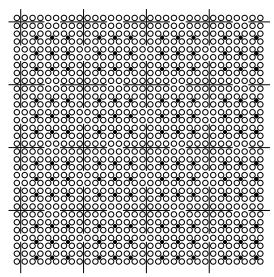

(b) Small crosses.

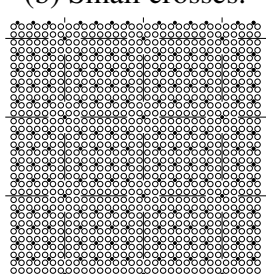

(d) Short lines.
Figure 6: Details of SU-8 structures in the chip's active area reducing the radial stress in the aluminium layer. The open circles represent the grid holes $(55 \mu \mathrm{m}$ pitch), the closed circles SU-8 pillars like in the current design. The crosses and lines in (a) and (c) have a length of $440 \mu \mathrm{m}$, the crosses and lines in (b) and (d) are $330 \mu \mathrm{m}$ long. The width for all cross and line structures is $17 \mu \mathrm{m}$.

Table 2: Overview of the groups of modified devices for thermal cycling. To improve the thermal homogeneity the carrier size was first chosen smaller and then exchanged by a metal block with cooling fins and heat-conductive paste. We tested the grid elasticity with glue applied to all grids in the third batch. In the end, only a small control group had Globtop on the grid edge.

\begin{tabular}{|c|c|c|c|}
\hline Batch & Samples & Designs & Chip carrier \\
\hline 1 & 20 & 10 & large Gel-Pak \\
\hline 2 & 8 & 4 (excl. R) & $\begin{array}{l}2 \text { small Gel-Pak } \\
\text { covers }\end{array}$ \\
\hline 3 & $\begin{array}{l}20 \text { (all with } \\
\text { Globtop) }\end{array}$ & 10 & $\begin{array}{l}\text { cooling block with } \\
\text { paste }\end{array}$ \\
\hline 4 & $\begin{array}{l}20 \text { (5 with } \\
\text { Globtop) }\end{array}$ & 10 & $\begin{array}{l}\text { cooling block with } \\
\text { paste }\end{array}$ \\
\hline
\end{tabular}

Reference [12] provides further details.

Two wafers of the modified samples were fabricated at Fraunhofer IZM to evaluate the differences between these patterns in terms of mechanical strength, thermal cycling robustness, and detector performance. The wafers have in common the silicon substrate, the SiRN protection layer of $2 \mu \mathrm{m}$ thickness, the SU-8 structures and the aluminium grid. One wafer, however, has an additional gold layer between the silicon substrate and the SiRN layer. This gold layer serves as anode to read out electrical signals.

\section{Thermal cycling experiments of modified SU-8 patterns}

Four batches of chip samples were tested in thermal cycles in a nitrogen gas atmosphere at temperatures down to $-130^{\circ} \mathrm{C}$. Table 2 compares the characteristics of all batches.

Judging from optical inspection, we have very uniform, nearly perfect grids. The adhesion between SU-8 and aluminium appears to be very good such that the grid as a layer is not torn apart. However, the devices in the first two batches 


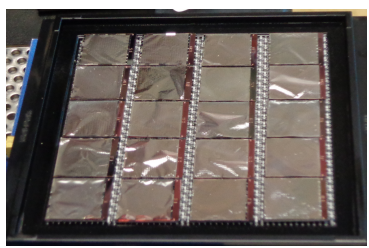

(a) First batch with 20 samples.

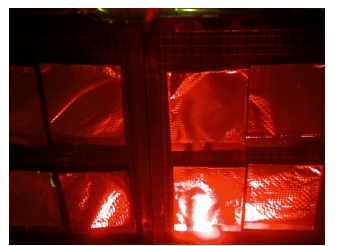

(b) Second batch with 8 samples.

Figure 7: Pictures of the first two batches after the respective thermal cycles. (a) shows the devices of the first batch after opening the vessel, (b) is a picture taken by the CCD camera of the second batch at the end of the cycle. Note that none of the actual grid layers is broken. In fact, the damage of the samples is the release of the SU-8 from the protection layer.

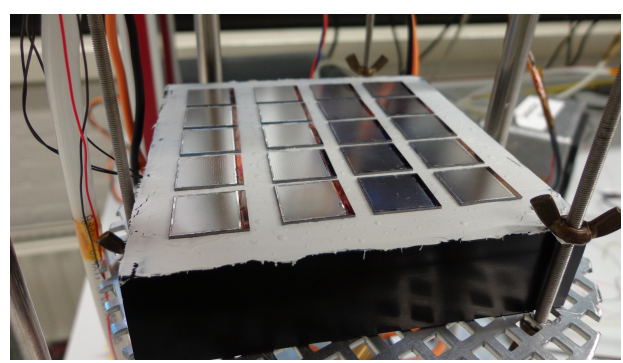

Figure 8: Picture of the third batch of samples after the thermal cycle. The fixation of the dyke with the help of glue as well as the improved thermal contact resulted in undamaged InGrids after this thermal cycle.

all got destroyed during the thermal cycle. The first of these ${ }^{160}$ devices showed the damage already at $-25^{\circ} \mathrm{C}$. All of the $28^{16}$ devices show the same kind of damage, see figure 7 parts of ${ }^{162}$ the ridges came off and/or the grid is wrinkled, despite using ${ }^{163}$ smaller chip carriers (and thus less devices) for the second batch ${ }^{164}$ to avoid thermal inhomogeneities among the devices.

When the InGrid structure is confronted with a cold sur- ${ }^{16}$ roundings, the grid can cool quicker than the silicon wafer, ${ }^{16}$ because the latter is much thicker and has lower thermal conductivity. A large temperature difference can then build up be- ${ }^{168}$ tween grid and chip, amplifying any thermal expansion prob- ${ }^{169}$ lems. Therefore a controlled cooldown requires the connec- ${ }^{170}$ tion of the silicon chip to a kind of heat sink, besides a low- ${ }^{17}$ enough cooling rate. This issue is addressed by mounting the ${ }^{172}$ samples on a metal plate with cooling fins with the help of heat- ${ }^{173}$ conductive paste.

To investigate the grid elasticity we glue the grid edges to the ${ }^{175}$ substrate using Globtop along the edges of a third batch of $20^{176}$ devices. These measures proved to be successful. As shown ${ }^{177}$ in figure 8 none of the 20 devices suffered any damage, neither ${ }^{178}$ the modified designs nor the reference devices with the original ${ }^{179}$ layout.

A final thermal cycle has to determine if the previous suc- ${ }^{18}$ cessful test is due to the improved thermal contact, i.e. smaller ${ }^{182}$ temperature gradients, the reinforced grid edges, or both. $\mathrm{We}^{183}$ selected 20 InGrid chips of 10 different designs (including again ${ }^{184}$ the reference InGrid). For better comparison with previous test ${ }^{185}$ results, the grid of a subset of 5 devices is fixated with glue. The ${ }^{186}$ outcome, as shown in figure 9 , gives a clear result: despite the good thermal contact all grids have delaminated, except for the

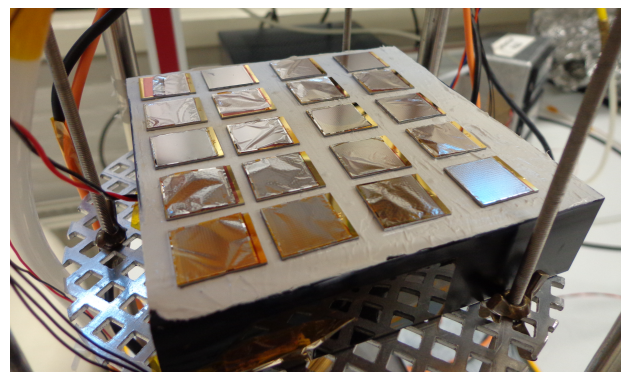

Figure 9: Picture of modified InGrids after the fourth thermal cycle. Although the grids as a layer are intact, the underlying SU-8 ridges have delaminated. Only the devices with reinforced grid edges are undamaged.

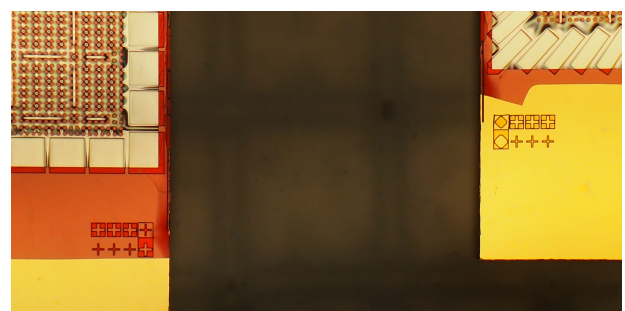

Figure 10: Microscope image of the SU-8 structures used for the shear test. These structures serve as alignment markers during the wafer post-processing. Note the SiRN layer (red) extending under the markers on the left chip. The adhesion there is a lot stronger than on the gold layer. Also visible are the different dyke layouts.

ones that were reinforced with glue. The aluminium layer of the grids itself, however, remained intact. Only the SU-8 edge supports delaminated from the SiRN, regardless of their layout.

A first conclusion is that the aluminium grid is ductile enough to sustain the whole thermal cycle. In addition to this, the heatconductive paste improved the thermal conditions, i.e. reduced any thermal gradient. Nevertheless, the SU- 8 dykes need to be fixed onto the chip edges by a thin film of glue.

\section{Adhesion strength}

With a shear test tool (Dage ShearTool 4000) it was investigated whether the thermal cycle leads to deterioration of the adhesion of SU-8 to the underlying SiRN layer. The samples of the fourth batch were subjected to a shear test before and after the thermal cycling.

For this test we use two groups of alignment markers on the "bond pad" area of each of the InGrid chips, each group consisting of 4 of these markers. Figure 10 shows an example of two chip corners with the alignment markers. For the adhesion test we use the 3 equal ones of each group. We measure the force required to delaminate the SU- 8 markers from the underlying substrate. On most of the chips the markers rest on the gold anode layer. On three devices the SiRN layer extends further on the "bond pad" area, see also figure 10 To exclude the possible influence of local variations across the wafer we perform the shear test on every chip.

We obtain results of the shear force measurements before and after the thermal cycle for 15 samples 1 The shear force re-

${ }^{1}$ Note that we exclude the 5 chips with Globtop around the grid edges. The 


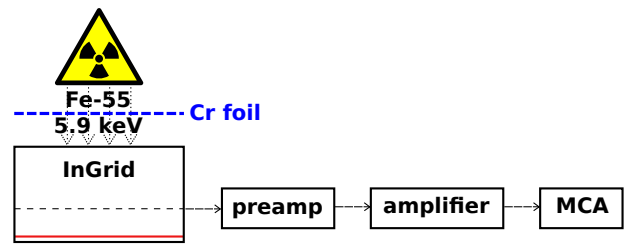

Figure 11: Schematic of the readout chain for the modified InGrid gaseous detector. A chromium foil largely absorbs the $K_{\beta}$ line of ${ }^{55} \mathrm{Fe}$ at $6.5 \mathrm{keV}$. The positive signal induced on the grid is shaped by the preamplifier and amplified by an Ortec 572 amplifier. An Amptek Pocket MCA8000A multi-channel analyzer records the pulse height spectrum of the signals. quired for delamination, as measured on 12 samples, amounts to $4.02(117) \mathrm{g}$ before, and 2.69(86) $\mathrm{g}$ after the thermal cycle. For SU-8 structures on SiRN, the values are 15.45(347) $\mathrm{g}$ and $7.95(481) \mathrm{g}$, respectively. We find that thermal cycling reduces the adhesion of SU-8 on its substrate significantly. In accordance with earlier studies [11], the results show a stronger adhesion between SU-8 and SiRN than between SU-8 and gold.

\section{Functional test of prototypes}

Although we use dummy chips rather than real pixel readout chips, the metal layer on the dummy substrate allows high voltage biasing of the structure to enforce gas gain. This allows the recording of signals by ionising radiation in a suitable gas, be it without position information. The pulse height spectrum of monochromatic X-rays from an ${ }^{55} \mathrm{Fe}$ source was recorded in this manner, using the setup shown in figure 11.

To this purpose the chips of three distinct layouts are glued on a PCB with contact lines and connection pins to connect the anode, the grid and the cathode to a power supply and the readout, respectively (see figure 12). The drift chamber consists of a thin glass-epoxy frame of $5 \mathrm{~mm}$ height, with gas tubes glued into one of its walls. Copper-cladded Kapton foil forms the cathode. A chromium foil between drift chamber and the source absorbs the $K_{\beta}$ line of ${ }^{55} \mathrm{Fe}$ at $6.5 \mathrm{keV}$. The gas tubes are connected to a flow controller supplying the $\mathrm{Ar} / \mathrm{iC}_{4} \mathrm{H}_{10} 90 / 10$ mixture.

The high voltage supplied to the grid is $V_{\text {grid }}=370 \mathrm{~V}$, and ${ }^{221}$ the drift field between grid and cathode is kept constant $\mathrm{at}^{222}$ $E_{\text {drift }}=300 \mathrm{~V} / \mathrm{cm}$. The anode is connected to ground poten- ${ }^{223}$ tial. The signal is read out via a high voltage pin, decoupled ${ }^{224}$ from the high voltage supply by a $100 \mathrm{nF}$ capacitor, and shaped $\mathrm{d}^{225}$ by a preamplifier. A closed metal box and ferrite rings around ${ }^{226}$ the signal cables minimise the pick-up noise. The gain of the ${ }^{227}$ amplifier is adjusted to use the full range of the ADC to record ${ }^{228}$ the pulse height spectrum of the signals.

The obtained spectra clearly show the photopeak at $5.9 \mathrm{keV}^{230}$ and the escape peak at $2.95 \mathrm{keV}$. See figure 13 for the spectra ${ }^{231}$ along with drawings of the SU-8 design of the respective sam- ${ }^{232}$ ple. To determine the energy resolution we fit a function that is ${ }^{233}$ artificially increasing the required shear force.

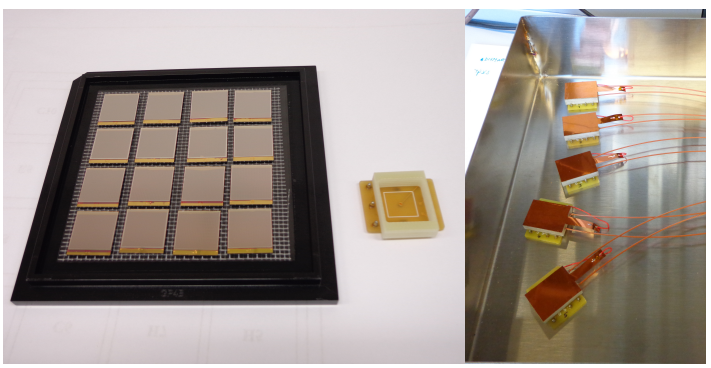

(a) PCB and frame for the drift (b) Assembled dechamber. tectors.

Figure 12: Pictures of the gaseous detectors built with modified InGrid chips as a readout.

Table 3: Energy resolution at the photopeak of ${ }^{55} \mathrm{Fe}(5.9 \mathrm{keV})$ of the tested modified InGrid designs.

\begin{tabular}{ccr}
\hline Sample & Characteristics & FWHM (\%) \\
\hline 1 & reference InGrid & $13.54(2)$ \\
2 & rectangles, crosses & $15.36(3)$ \\
3 & rectangles, lines & $14.06(2)$ \\
\hline
\end{tabular}

the sum of three Gaussians and a linear function

$$
\begin{aligned}
f(x)=\underbrace{A \exp \left(\frac{\left(x-\mu_{e}\right)^{2}}{2 \sigma_{e}^{2}}\right)}_{\text {escape peak }}+\underbrace{B \exp \left(\frac{\left(x-\mu_{p}\right)^{2}}{2 \sigma_{p}^{2}}\right)}_{\text {photopeak, } K_{\alpha}} \\
+\underbrace{C \exp \left(\frac{\left(x-\mu_{\beta}\right)^{2}}{2 \sigma_{\beta}^{2}}\right)}_{\text {photopeak, } K_{\beta}}+D+E x .
\end{aligned}
$$

The third Gaussian is for taking into account the suppressed (but remaining) $K_{\beta}$ line of ${ }^{55} \mathrm{Fe}$ at $6.5 \mathrm{keV}$. The peak position of the third Gaussian $\mu_{\beta}$ is not a free parameter, but fixed with respect to $\mu_{p}-\mu_{e}$. From the standard deviation of the fit we determine the FWHM according to

$$
\mathrm{FWHM}=2 \ln 2 \times \sigma_{p} \approx 2.35 \sigma_{p} .
$$

Table 3 compares the FWHM of the spectra of the different samples. The SU-8 design with the best energy resolution is the reference layout, followed by the design with lines before the one that has crosses next to the pillars. We can conclude that additional SU-8 structures influence the detector's energy resolution where cross structures disturb the avalanches more than lines.

To determine the reason for the loss in energy resolution we record a multitude of pulse height spectra in short consecution. For this purpose we use the the detector with sample 2 where we found the worst energy resolution since any effect will be most visible with that detector. This measurement is divided into three time series. The second series was taken after leaving the high voltage off for about 5 hours. The third series started on the following day.

We find that the energy resolution is not time-dependent: apart from a small decrease during the first five minutes it does 


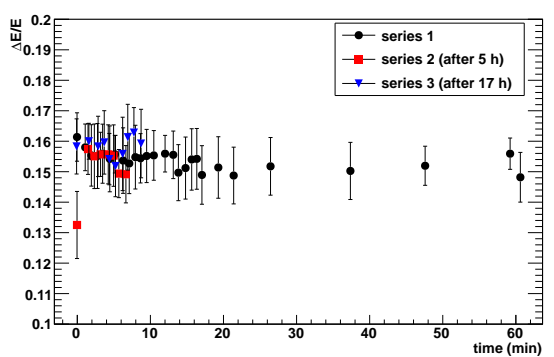

(a) Energy resolution at the $5.9 \mathrm{keV}$ photopeak.

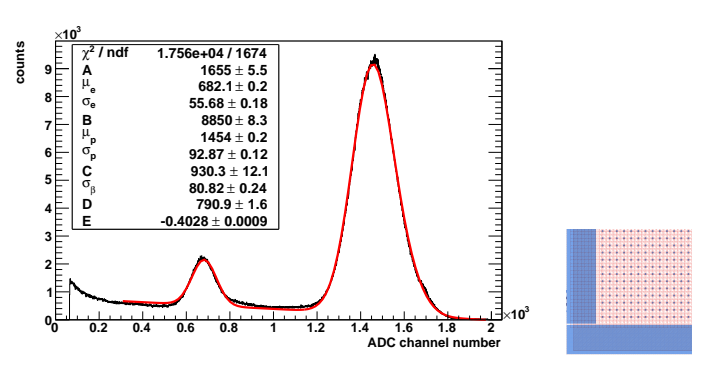

(a) Sample 1 .

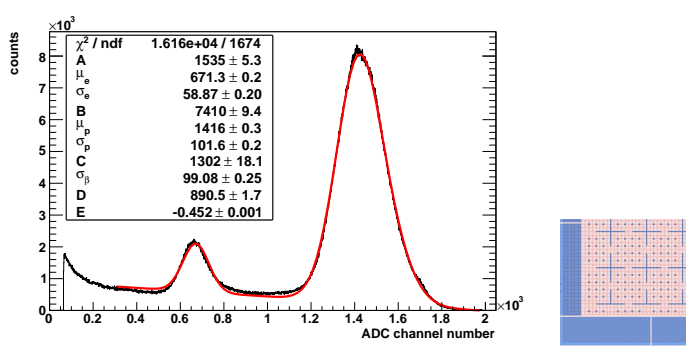

(b) Sample 2 .

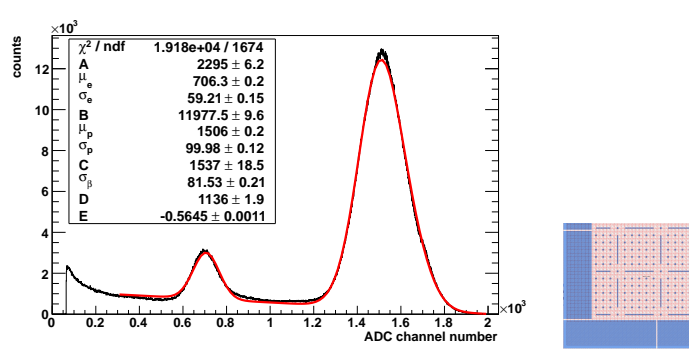

(c) Sample 3.

Figure 13: Pulse height spectra of an ${ }^{55} \mathrm{Fe}$ source recorded with prototype detectors in $\mathrm{Ar} / \mathrm{C}_{4} \mathrm{H}_{10}$ 90/10. Note that the ADC threshold is chosen higher than the baseline. Moreover, the DC level of the amplifier's output is slightly neg- ${ }^{247}$ ative. This is compensated by using the known relative distance between the ${ }^{248}$ photo- and the escape peak. Next to the spectra the images show details of the249 respective SU-8 design.

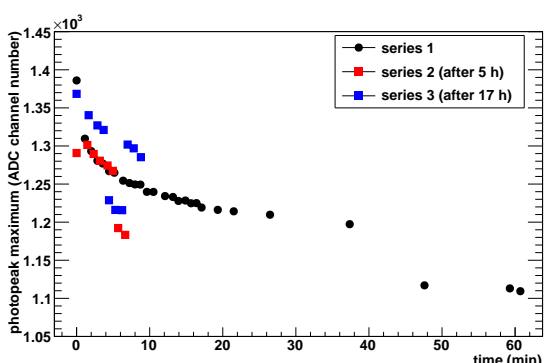

(b) Photopeak position. Error bars are smaller than the markers.

Figure 14: Time dependence of the energy resolution and peak position. While the energy resolution does not decrease notably, the position of the photopeak (in ADC channels) shift towards lower values, so less charge is being collected. This indicates that parts of the avalanche being quenched by charged-up SU-8 structures. The second and third time series were taken after breaks of 5 and 17 hours, respectively. They show the same initial behaviour of a yet uncharged detector.

not show a significant trend. We do observe, however, that the position of the photopeak moves to lower values in terms of ADC channels, see figure 14 . The pulse height hence decreases over time, meaning that less charge is moving within the amplification region (in form of an avalanche). The subsequent series show a similar behaviour for the initial period when the SU-8 of the detector is not yet charged up. This indicates that the SU-8 is indeed charging up over time.

\section{Conclusions}

GridPix emulating test samples were successfully cooled down to $-130^{\circ} \mathrm{C}$. A low cooling rate and thermal contact with the environment were shown to be of critical importance. Only Globtop-reinforced grids provide the desired stability of the GridPix structure. This cannot be achieved through a dedicated SU-8 pattern alone.

Instead the special reinforcing SU-8 structures tested in this work have a negative influence on the energy resolution of the GridPix detector; and having no decisive effect on the mechanical stability, they are better avoided.

\section{Acknowledgements}

We like to thank Richard Rosing for the FEM simulations. 


\section{References}

[1] V.M. Blanco Carballo et al., IEEE Electron Device Letters 29 (6) (2008), 585-587.

262 [2] Y. Giomataris et al., Nucl. Instr. and Meth. A 376 (1) (1996), 29-35.

[3] X. Llopart et al., IEEE Transitions on Nuclear Science 49 I (5) (2002), 2279-2283.

265 [4] X. Llopart et al., Nucl. Instr. and Meth. A 581 (2007), 485-494.

266 [5] M. Chefdeville et al., Nucl. Instr. and Meth. A 556 (2) (2006), 490-494.

267 [6] W.J.C. Koppert et al., Nucl. Instr. and Meth. A 732 (2013), 245-249.

268 [7] R. Schön et al., Nucl. Instr. and Meth. A 718 (2013), 446-449.

269 [8] L. Baudis for the DARWIN consortium, Dark Matter Wimp Search with Noble Liquids, 2010, arXiv:1012.4764.

271 [9] R. Schön et al., to be published in Nucl. Instr. and Meth. A, doi:10.1016/j.nima.2014.10.063.

273 [10] S. Bystrova et al., Microelectronic Engineering 84 (5-8) (2007), 11131116.

275 [11] V.M. Blanco Carballo et al., Microelectronic Engineering 86 (2009), 765768.

[12] R. Schön, Development of liquid xenon detector technology for dark matter searches, PhD thesis under preparation, Universiteit van Amsterdam. 